\title{
Numerical Receding Contact Analysis of a Perfect-Fit Pin and Bushing in a Loaded Plate
}

\author{
Branimir RONČEVIĆ, Ante BAKIĆ, Janoš KODVANJ
}

\begin{abstract}
Numerical analysis is carried out for a frictionless receding contact problem of a perfect-fit pin and bushing in a uniaxially loaded plate. The problem is analysed using the finite element method under the assumption of plane strain and linear elasticity. The problem is investigated for the influence of external load and for different geometries. The obtained contact pressures show uncharacteristic behaviour, where higher peak values occur on contact surfaces with larger contact angles. This seemingly contradicts not only the physics of receding contact problems, but also the physical laws of contact problems in general. It is shown that the reason for this unusual behaviour is, in fact, in the physical reduction of the contact area as the radius of curvature decreases. This class of problems shows to be linearly dependent on the intensity of load as regards the contact stresses, and load has no influence on the contact angles.
\end{abstract}

Keywords: finite element method; loaded plate; perfect-fit pin and bushing in a plate; receding contact problem

\section{INTRODUCTION}

Most problems in contact mechanics follow the physically intuitive principle of an increasing contact area with the increase of load. In contrast to that, in a separate class of conforming contact problems the contact area shrinks with the application of load, and such contacts are referred to as receding contacts. In the strictest sense, as per definition given by $\mathrm{K}$. L. Johnson, a receding contact is one in which the loaded contact area is completely contained within the unloaded contact area [1]. Receding contacts typically occur in structural problems that involve unbonded layers pressed into a foundation and in problems that can be reduced to the case of a perfect-fit or interferencefit pin-plate connection. Generally speaking, the problem of separation (i.e. of receding of the contact area) between a pin and a plate can be studied for the case of a loaded pin, loaded plate and a combination of both cases.

The pin-plate problem, which is akin to the subject of interest in this paper, is encountered in a multitude of connections that employ bolts, pins or rivets as joining elements. Pin-plate connections received much attention in the scientific community primarily in the context of aviation industry, where rivet connections and bolts are used in sheet assembly of the outer skin of an aircraft. However, the design of reliable attachments and joints is of the utmost importance in every niche of transportation technology and general machinery, since structural failures are very likely to occur at or in the vicinity of joints, which must always be understood as the primary sources of weakness in any structure.

Some of the older analytical studies dealing with receding contacts for the pin-plate problem were done by Stippes, Wilson and Krull [2], Noble and Hussain [3] and Margetson and Morland [4]. A reference to a comprehensive series of photoelastic measurements on the subject is also given in [4]. An experimental method based on photoelastic method for continuous tracking of the separation between the pin and the plate is formulated by Ghosh, Dattaguru and Rao in [5]. Both analytical and numerical solutions are given by Mangalgiri et al. in [6] for the cases of loaded pin, biaxially loaded plate and the combination of the two. All these studies were done within the frame of linear theory of elasticity, the problem was considered as frictionless and the pin was assumed as rigid. Man used the boundary element method in [7] to investigate the cases of a loaded pin and a loaded plate, taking into account the influence of friction as well. With the development of computers and various numerical methods the scope of interest widened toward modelling of crack propagation and effects of plasticity. Satish Kumar et al. used FEM in [8] for the elastic-plastic analysis of interference-fit connections with the aim of determining the lifespan of joints subjected to cyclic loads.

The subject of composite (laminates and fibre-reinforced materials), anisotropic and orthotropic materials for the plate also received significant attention in literature. A very comprehensive study by de Jong in his $\mathrm{PhD}$ thesis [9] can be singled out. Murthy et al. [10] analyse the stress field and the load carrying capacity of joints within anisotropic laminated materials and Camanho and Matthews [11] give a systematic literature review on stress and load carrying analysis of joints in fibre-reinforced plastics.

Ciavarella and Decuzzi formulated analytical solutions taking into account the elasticity of the pin, in [12] for the case of material similarity and in [13] for the case of material dissimilarity. Their studies were limited to the frictionless case of loaded pin. Their most important finding is the existence of the contact angle $\varphi_{\lim }$ that corresponds to the case of a perfect-fit, but which also represents the asymptotic solution for both clearance- and interference-fit joints. Ho and Chau [14] developed an analytical model that accounts for both material dissimilarity and friction, but the solutions are not very accurate because the contact angle is assumed in advance to be $90^{\circ}$. More accurate results were obtained in [12] and [13] and by Iyer in [15], who conducted a thorough FEM analysis of a perfect-fit case for finite and infinite plate, uniaxial and biaxial plate loading, presence of friction and material dissimilarity. Hou and Hills gave a significant contribution in the mathematical treatment of the problem in [16-18]. Ciavarella et al. [19] investigated by means of a FEM analysis the behaviour of contact parameters for the case of loaded pin in a conrodbushing-gudgeon pin assembly in correlation to the type of fit between the elements. Here, they introduced the terms of progressive (clearance-fit) and regressive (interference-fit) contact and found that the contact angle converges to the same value in both cases.

The present paper is also aimed at the more complex structural problem of a cylindrical pin in perfect fit with a 
bushing, which is in perfect fit with a hole in a plate. The problem is modelled under plane strain assumption for the case of a loaded plate and is assumed to be frictionless. From this follows that only compressive tractions can be transmitted on the contacting surfaces. All bodies in contact (pin, bushing and plate) are assumed to be elastic and isotropic. In Chapter 2 a brief description of the problem and of the numerical model is given, along with an outline of Femap software in the context of contact modelling. In Chapter 3 numerical results are presented and discussed and, finally, in Chapter 4 a short comment on the obtained results is given and complemented by an outlook to future research.

\section{DESCRIPTION OF THE PROBLEM AND NUMERICAL MODEL}

\subsection{Description of the Problem}

The case of a receding contact between a pin, bushing and a plate, as shown in Fig. 1, to the best of the authors' knowledge has not received significant attention in literature as a separate object of scientific interest. Despite that, there is a practical value to this field of study - bushings are often used as repair elements in various sorts of non- permanent joints, and also as integral parts of the intended design in various mechanical links and joints. For instance, bushings are regularly used with fully-floating gudgeon pins in connections between pistons and connection rods in internal combustion engines. As already stated in the introduction, a rare reference to this aspect of the problem was made by Ciavarella et al. in [19].

When the plate is not loaded, the contact at the platebushing interface is realized over the whole circumference, i.e. the total contact angle equals $\theta_{1}=2 \pi$. The same applies to the total contact angle $\theta_{2}$ at the pin-bushing interface. Since the problem is considered as a theoretical perfect-fit, no tractions exist at the interfaces of the bodies before the plate is loaded. Once the plate is loaded, partial separation on the contact surfaces is ensued by the deformation of the plate, producing at the same time compressive forces (contact pressure) $p_{\mathrm{n} 1}$ on the bushing over the area where separation did not take place $\left(\theta_{1}<2 \pi\right)$. These compressive forces now deform the bushing, so separation also takes place between the bushing and the pin. This finally produces contact pressures $p_{\mathrm{n} 2}$ acting over the remaining total contact angle $\theta_{2}<2 \pi$ between the bushing and the pin.

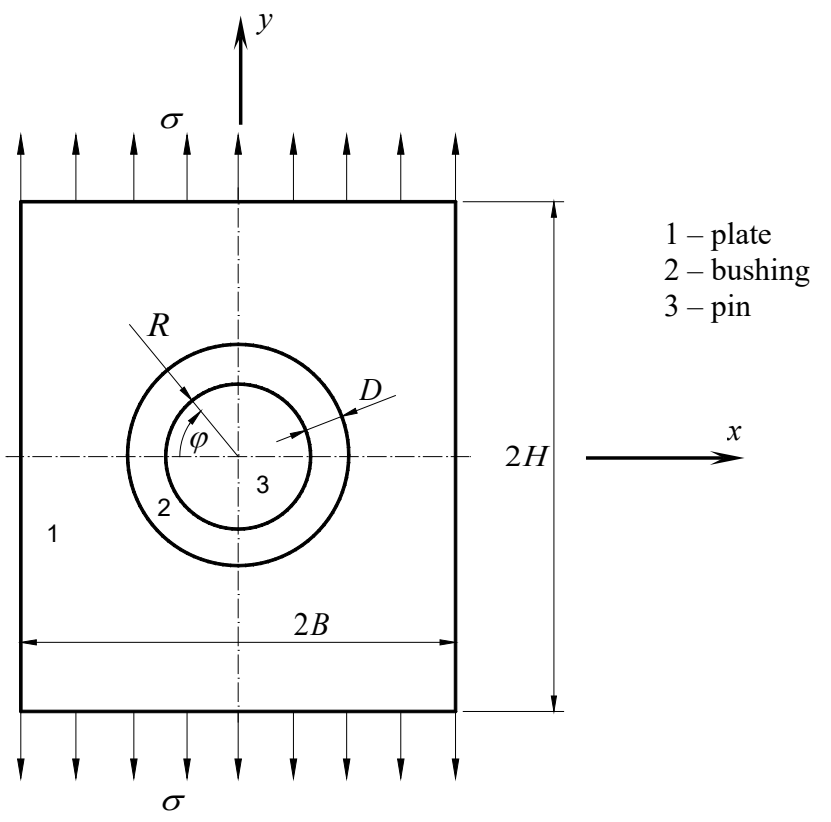

Figure 1 The problem of a pin inside a bushing, which is located in a hole of a plate uniformly loaded in tension

The primary unknowns in this problem are the extents of both contact surfaces (total contact angles) and the corresponding values of maximal contact pressures. It must be noted that the total contact angle $\theta_{1 / 2}$ is discontinuous on both contact surfaces. Because of the central symmetry of the problem, the contact angles to the left and to the right of the $y$ axis amount to one half of the total contact angle:

$2 \varphi_{1 / 2}=\frac{1}{2} \theta_{1 / 2}<\pi$

These angles are also distributed symmetrically about the $x$-axis and it is, therefore, sufficient to determine just one quarter of both total contact angles. In accordance with Eq. (1), these angles are denoted as $\varphi_{1 / 2}$.

\subsection{Numerical Model}

The material for all three contacting bodies is defined as isotropic and linearly elastic and the model is solved using nonlinear static analysis in the software package Femap. Material properties for all three bodies are $E=200$ GPa and $v=0.3$ and the model is meshed with plane strain finite elements. Tension load acting on the plate is defined as a uniformly distributed stress in the $y$-direction $\sigma=10^{8}$ $\mathrm{N} / \mathrm{mm}$.

The problem shown in Fig. 1 is investigated from two aspects: (i) the influence of load intensity and (ii) the effect of the bushing thickness $D$ on the parameters of contact. Hence, four different geometries are analysed to account for the differing geometry, with the ratio $D / R$ (see Fig. 1) as the only parameter varying over the set of values $D / R=$ 
$0.1 ; 0.2 ; 0.5$ and 1. All other dimensional quantities and relations are kept constant in all the models: $H / B=2, B / R$ $=10$ and $R=1$.

Only the left half of the structure shown in Fig. 1 is modelled in Femap and a detail of the mesh in the vicinity of the hole is shown in Fig. 2. The plate and the pin are divided into regions for the purpose of uniform structuring of the mesh in order to ensure good accuracy of the result in the region of primary interest.

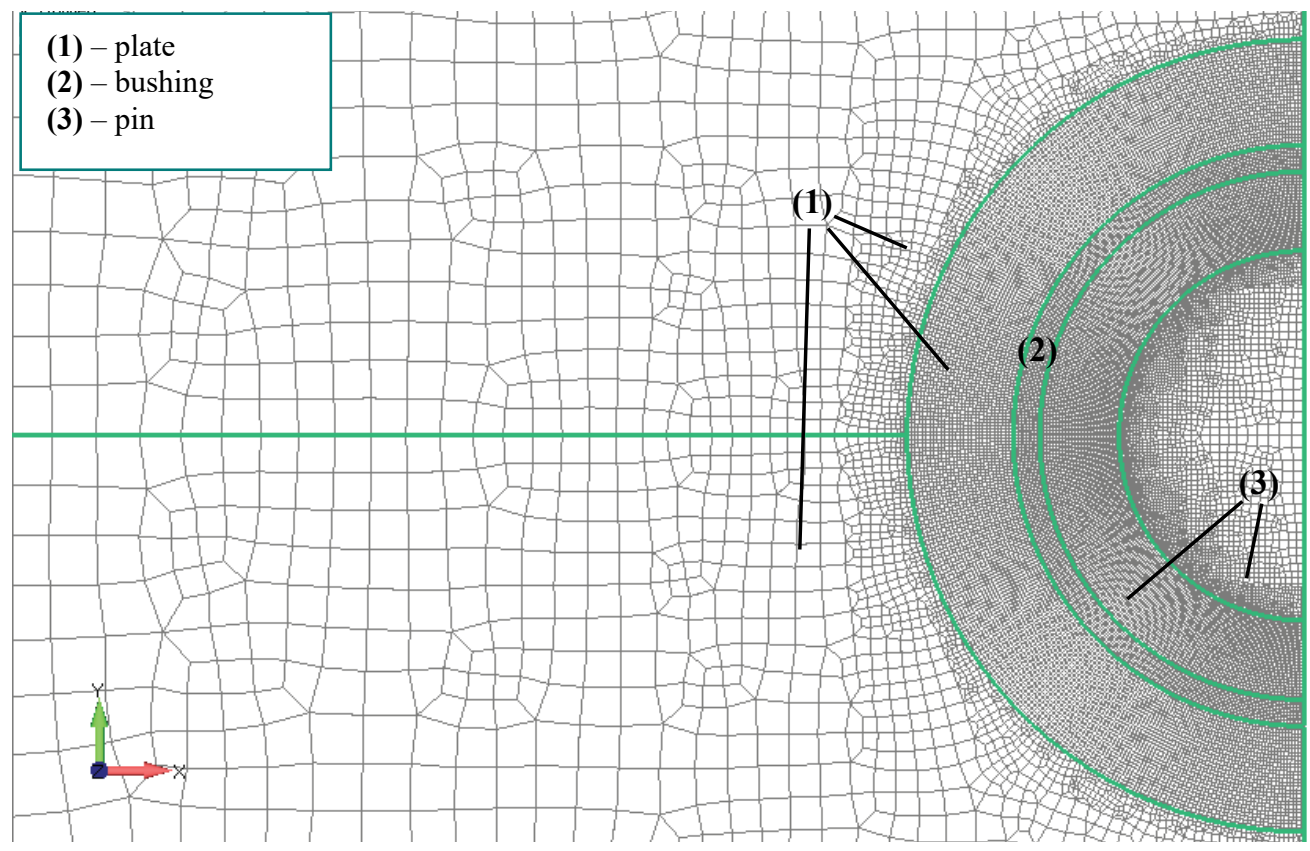

Figure 2 Mesh detail in the vicinity of the hole for $D / R=0.1$

The transmission of forces between the three bodies shown in Fig. 2 is modelled using slide line elements, which offer significant advantages over the limiting capabilities of now quite outdated gap elements. Their primary advantage is the capability to deal with non-conforming meshes (i.e. node-on-element), but they also allow large displacements of the contacting surfaces in the tangential direction (i.e. along the slide line).

A slide line element can contain an arbitrary number of nodes that are lying on the lines or curves of both bodies where contact forces are transmitted or are expected to take place with the application of load. The nodes of one body can be designated as either master nodes or slave nodes, and each slide line element contains a set of master nodes on one body and slave nodes on the other. For the purpose of this study two slide line elements are defined - one between the plate and the bushing, and another between the bushing and the pin. The nodes on the circular contour of the plate were taken as the slave nodes and on the outer contour of the bushing as the master nodes. Conversely, nodes on the inner contour of the bushing were taken as the slave nodes and on the circular contour of the pin as the master nodes. A three-node (two master nodes $M_{1}, M_{2}$ and one slave node $S$ ) slide line element is shown in Fig. 3.

The line segments joining two neighbouring slave or master nodes are called the slave or master segments. By default, the contact algorithm of Femap/Nastran checks the slave nodes for penetration into the master segments (as shown in Fig. 3), which is referred to as unsymmetrical penetration. However, both slave and master nodes can be checked for penetration if better accuracy of the result is sought, which is then referred to as symmetric penetration $[20,21]$. The algorithm uses the penalty method in imposing the displacement compatibility conditions and for the calculation of the contact forces.

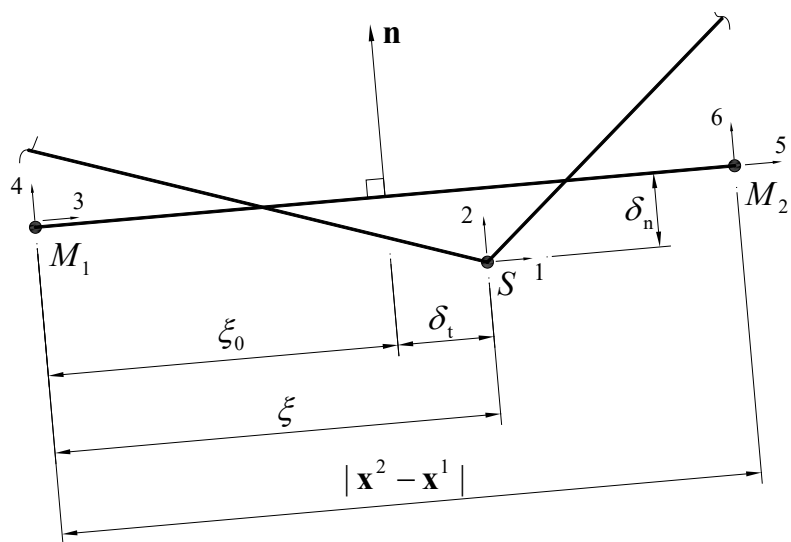

Figure 3 Three-node slide line element: $\xi, \xi_{0}-$ current and previous surface coordinate, $\delta_{\mathrm{n}}$ - penetration of slave node into the master segment, $\delta_{\mathrm{t}}$ - sliding of the slave node on the master segment

\section{ANALYSIS RESULTS AND DISCUSSION}

\subsection{Transmission of Contact Forces}

The distributions of contact pressures $p_{\mathrm{n} 1}$ at the platebushing interface and $p_{\mathrm{n} 2}$ at the bushing-pin interface correspond to the nodal values of the radial stresses $\sigma_{\mathrm{r}}$ (polar coordinates) from the FEM model. For the geometry with $D / R=0.1$ they are shown in Fig. 4 in dimensionless form.

Based on the diagrams in Fig. 4 an interesting observation can be made. Both the maximal contact pressure and the contact angle can be greater on the bushing-pin contact surface in comparison to the plate-bushing contact surface, although external load is exerted on the contact surfaces in the opposite order. This seemingly makes no physical sense for conforming contacts, especially if this 
result is compared to the conventional results for receding contacts in layered structures, for example in [22-24]. As a general rule, an external load $P$ transmitted over a larger

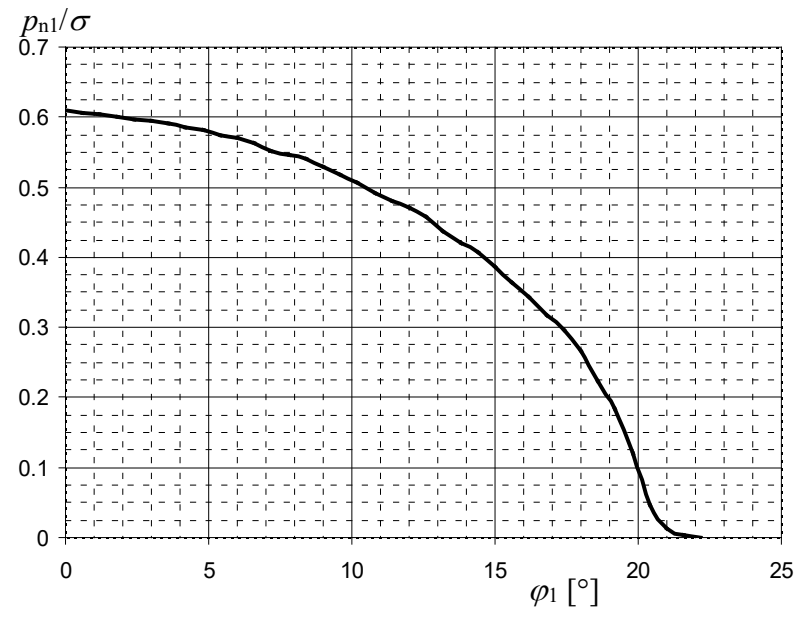

a) contact area always implies smaller maximal (and average) contact pressures and vice versa, otherwise the system would not be in a state of static equilibrium.

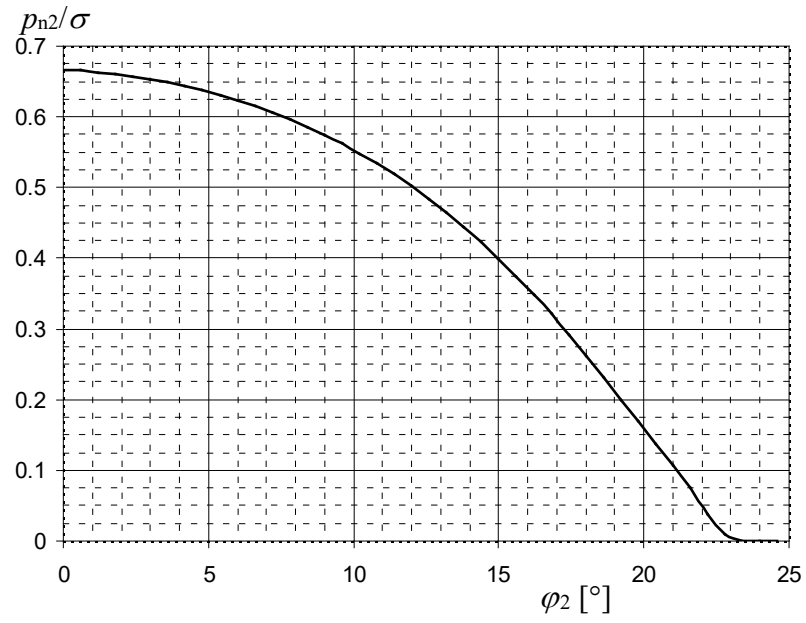

b)

Figure 4 Contact pressures: a) plate-bushing interface; $p_{\mathrm{n} 1, \max } / \sigma \approx 0.61, \varphi_{1} \approx 22^{\circ}$, b) bushing-pin interface; $p_{\mathrm{n} 2, \max } / \sigma \approx 0.666, \varphi_{2} \approx 23.4^{\circ}$

The following polar equation of static equilibrium must hold for two contact surfaces separated by their radial distance $D=r_{1}-r_{2}$ :

$$
\int_{-\varphi_{1}}^{\varphi_{1}} p_{\mathrm{n} 1}(\varphi) r_{1} \mathrm{~d} \varphi=\int_{-\varphi_{2}}^{\varphi_{2}} p_{\mathrm{n} 2}(\varphi) r_{2} \mathrm{~d} \varphi
$$

where $r_{1 / 2}$ denotes the radius of the contact surface. Since contact forces are transmitted over an arc length equal to $\int r \mathrm{~d} \varphi$, for a given difference of contact angles the physical extent of the bushing-pin contact surface can be smaller than that of the plate-bushing contact surface if $D$ is sufficiently large. In such a case static equilibrium dictates that the stresses must rise if the overall load were transmitted over a reduced surface area. Based on these considerations, and using Eq. (1), the following mathematical relations can now be formulated:

$$
\int_{-\varphi_{1}}^{\varphi_{1}} r_{1} \mathrm{~d} \varphi>\int_{-\varphi_{2}}^{\varphi_{2}} r_{2} \mathrm{~d} \varphi \Rightarrow p_{\mathrm{n} 1, \max }<p_{\mathrm{n} 2, \max },
$$

which proves that the mechanical principle of the obtained result is sound. It can, therefore, be misleading to estimate the contact surface in a "layered-type" centrally symmetric receding contact solely on the calculation of the contact angles. Even for a qualitative assessment of the relations between the contact stresses, true values for surface areas of the contact interfaces must be known.

\subsection{Influence of Load Intensity and Bushing Thickness}

Each geometry $D / R$ was investigated for a sequence of gradually increasing loads $\sigma_{\mathrm{gr}}$ of intensities $0.2 \sigma ; 0.4 \sigma$; $0.6 \sigma ; 0.8 \sigma$ and $\sigma$. Figures $5-8$ show the corresponding contact pressure distributions for all geometries.

It is evident that the diagrams are qualitatively similar. Consolidating all the results for maximal contact pressures and contact angles for all $D / R$ geometries with respect to load intensity gives the diagrams shown in Figs. 9 and 10.

A closer investigation of Figs. 9 and 10 suggests that the problem of pin and bushing inside a plate loaded in tension is a strongly linear receding contact problem, which behaves in almost perfect conformity with classical assumptions and simplifications for receding contacts that involve indentation of elastic layers into a substrate. The conventional approach in these cases was to regard the extent of the contact area as independent of the level of loading, while the contact stresses were understood to be linearly dependable on load intensity [1, 22-24]. The same assumptions also apply to a regular pin-plate contact problem. The study by the authors in [25] has to a great degree refuted the soundness of these assumptions for the problem of layer indentation with an elastic indenter, where the problem proved to be nonlinear. However, in this case the structural behaviour is shown to be completely analogous to the classical linear case of receding contacts.

As regards the influence of bushing thickness $D$ (i.e. the ratio $D / R$ ) on the parameters of contact, there seems to be no clear tendency for the maximal contact pressures, whose values for different geometries are grouped closely together, especially for the bushing-pin interface. It can be inferred that this small scatter of contact pressure values is also influenced by numerical error, but such an inference should be additionally scrutinized in future research. For the contact angles, it can be concluded that the bushing thickness greatly influences the contact angle at the bushing-pin interface (Fig. 10b), while at the same time having a very limited effect on the contact angle at the plate-bushing interface (Fig. 10a). The mechanical principle behind this behaviour is, again, linked to the fact that the overall plate-bushing contact surface increases with the outer radius of the bushing. This strongly influences the contact angles on the pin, which for thicker bushings leads to very little separation between the bushing and the pin. This phenomenon is evident from Fig. 10b, where $\varphi_{2}$ gradually approaches $40^{\circ}$. 


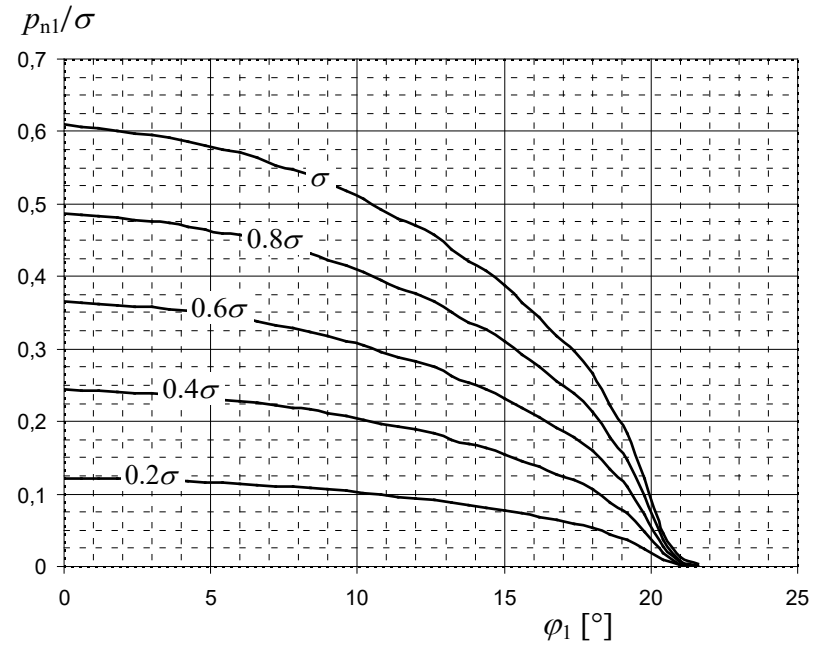

a)

Figure 5 Distributions of contact stresses for different load intensities for geometry $D / R=0.1$ : a) plate-bushing interface, b) bushing-pin interface

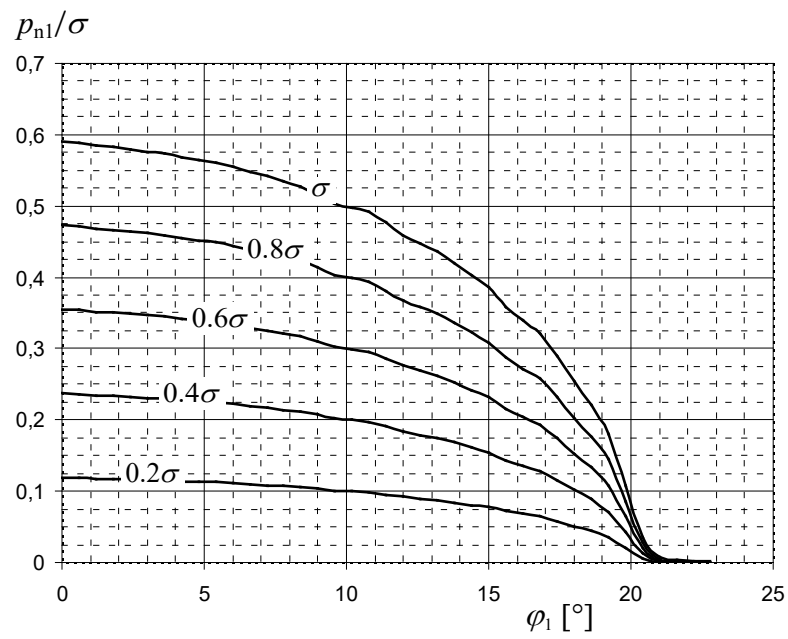

a)

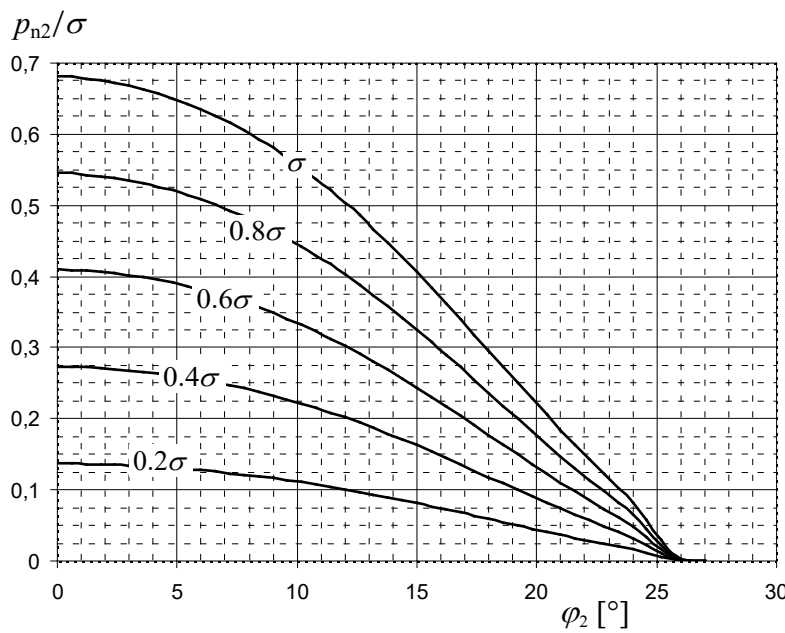

b)

Figure 6 Distributions of contact stresses for different load intensities for geometry $D / R=0.2$ : a) plate-bushing interface, b) bushing-pin interface

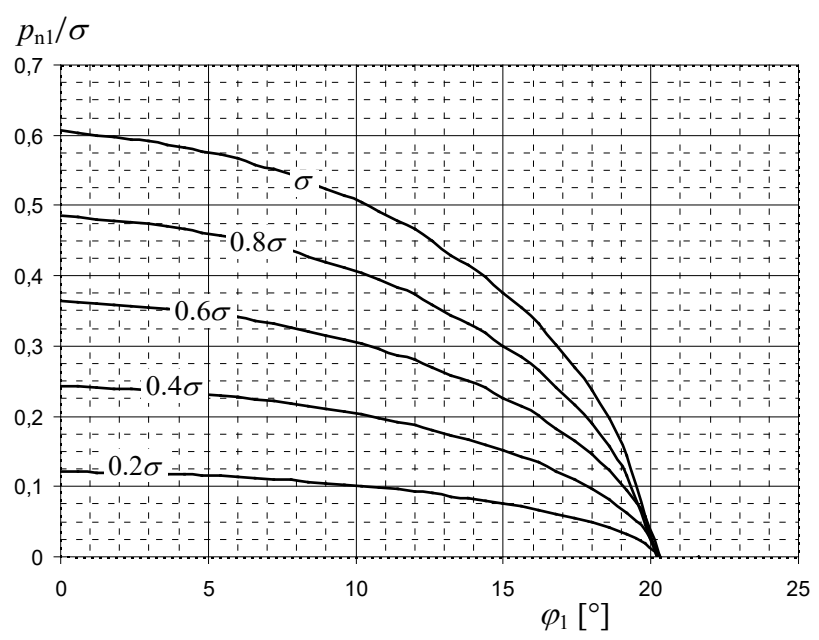

a)

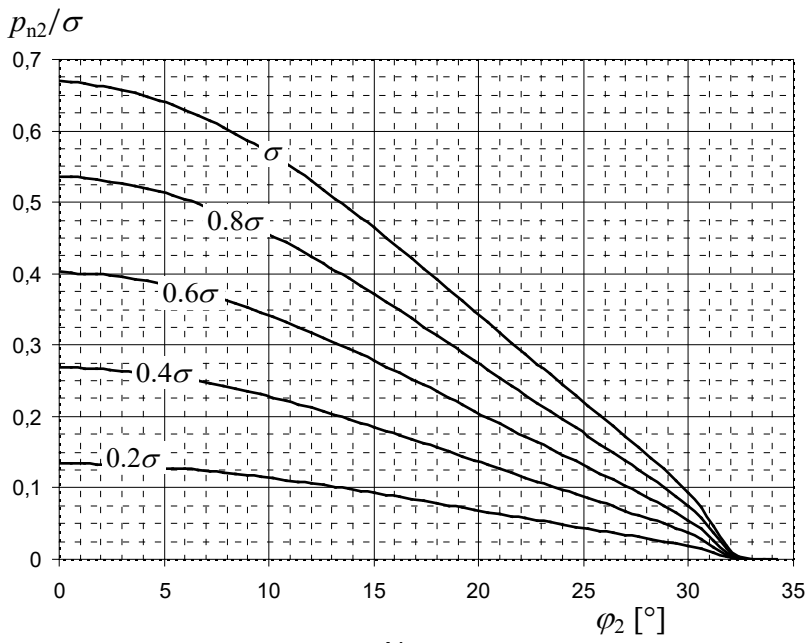

b)

Figure 7 Distributions of contact stresses for different load intensities for geometry $D / R=0.5$ : a) plate-bushing interface, b) bushing-pin interface 


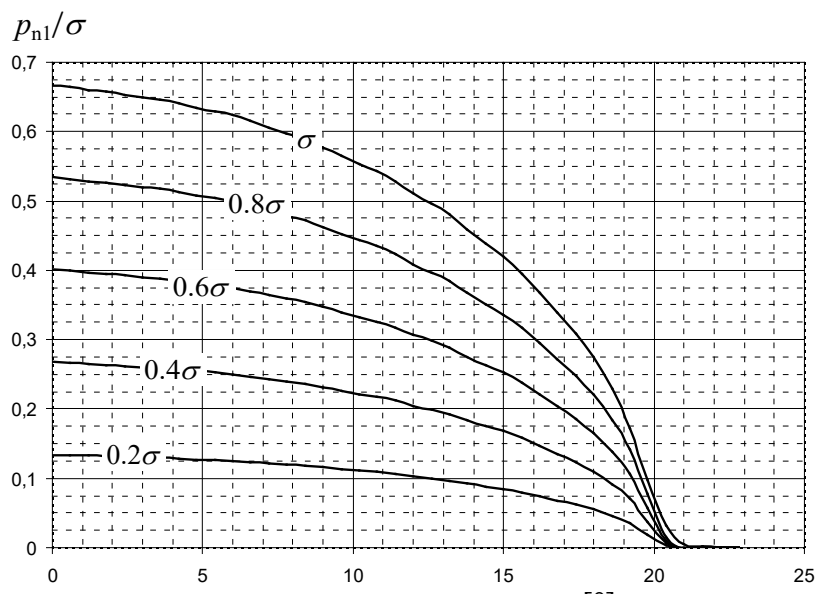

$\varphi_{1}\left[^{\circ}\right]$

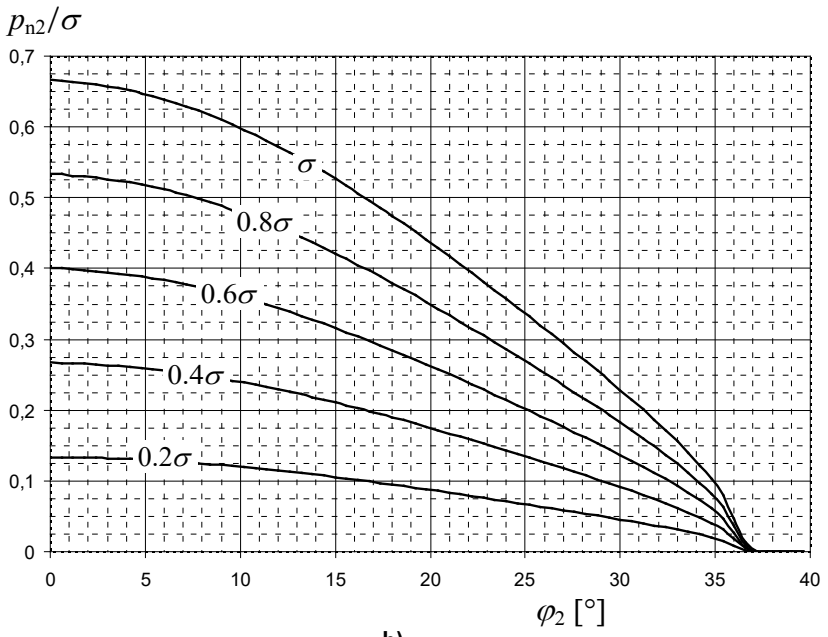

b)

Figure 8 Distributions of contact stresses for different load intensities for geometry $D / R=1$ : a) plate-bushing interface, b) bushing-pin interface

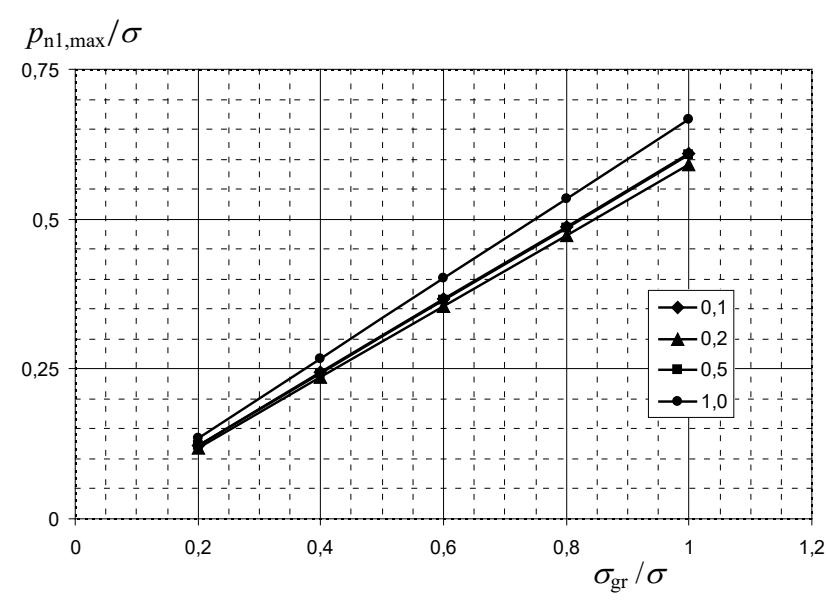

a)

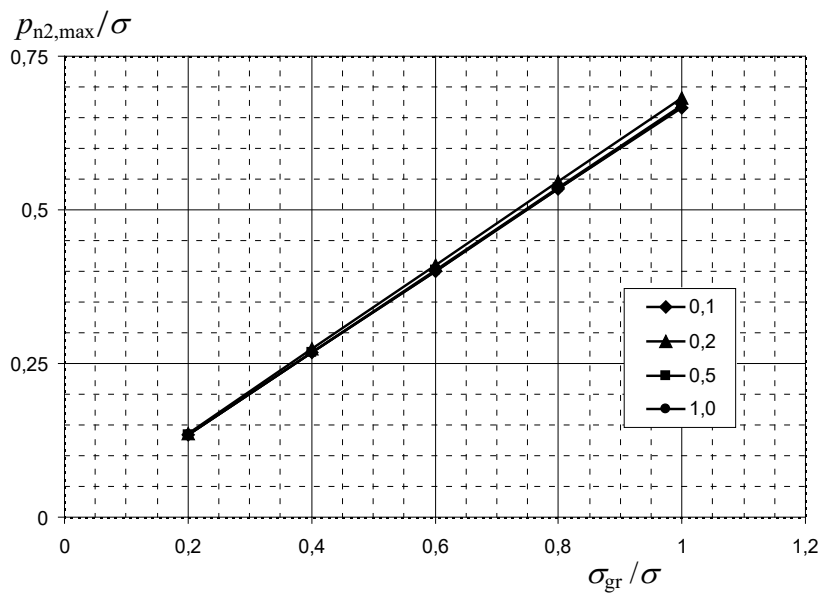

b)

Figure 9 Variation of maximal contact pressures with load intensity for all $D / R$ geometries; a) plate-bushing interface, b) bushing-pin interface

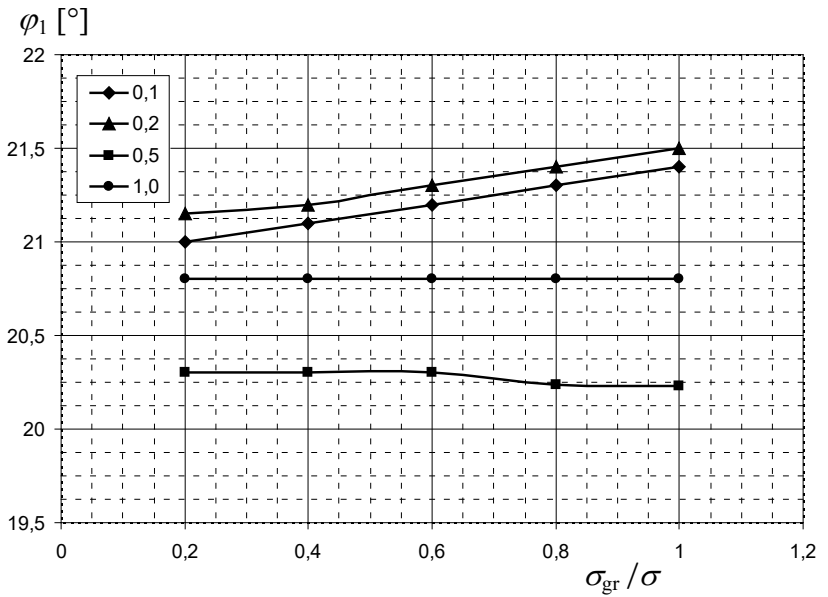

a) $\varphi_{2}\left[^{\circ}\right]$

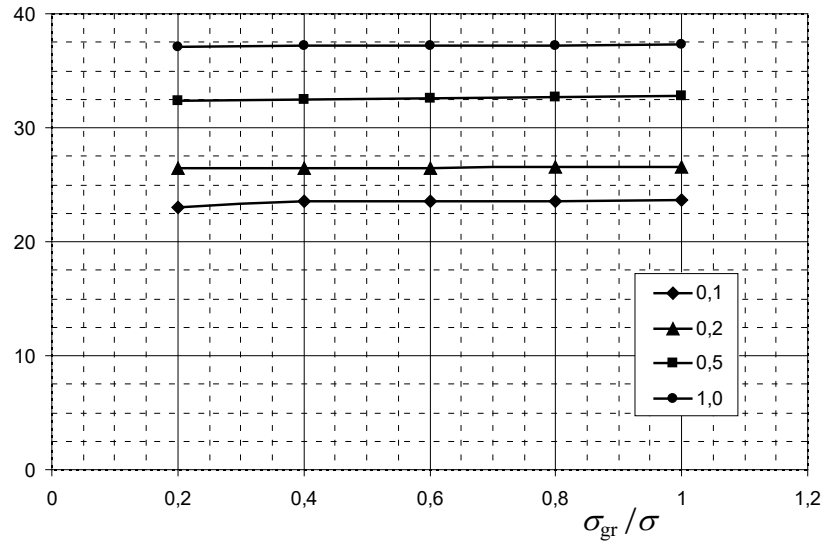

b)

Figure 10 Variation of contact angles with load intensity for all $D / R$ geometries; a) plate-bushing interface, b) bushing-pin interface

One very significant aspect of the problem that merits explanation is the fact that maximal contact pressures for a given load level change very little (or stay almost the same) with the increase of the contact area (as $D$ is increased). This inevitably implies that the overall load exerted on the contact surface also increases in approximately the same proportion. Since the plate is uniaxially loaded in tension in the $y$ direction (see Fig. 1), all points except those on the $y$ axis undergo a displacement in the $x$ direction as well, due to lateral strain. It is the constraint of this movement caused by the presence of the bushing and the pin that actually produces the contact pressures, which are 
proportional to the magnitude of displacement that they are constraining. The $x$-displacements change as a function of the $x$ coordinate, with points on the outer edge of the plate undergoing maximal $x$-displacements, while points lying on the $y$ axis not moving in the $x$ direction at all. For that reason the effective force that acts on the bushing changes with the outer diameter of the bushing.

\section{CONCLUSION AND OUTLOOK}

Contact parameters of a receding contact of a perfectfit pin and bushing in a loaded plate were investigated for the case of different load levels and different geometries, with the thickness $D$ of the bushing as the only variable quantity. Based on the presented results the following can be concluded:

- The influence of load level is very much in agreement with conventional assumptions of receding contacts; i.e. the contact area is independent of the level of loading and the contact stresses are linearly dependable on the intensity of external load.

- The bushing thickness has a noticeable impact on the contact angle for the bushing-pin interface, but in other aspects the influence is not very pronounced.

- The most interesting contribution of this work is the discovery of the intricate influence the bushing thickness exerts on the contact parameters - although the contact angle $\varphi_{1}$ does not seem to change significantly, the surface area of the plate-bushing interface does, in fact, change very significantly. However, this is also accompanied by the corresponding increase of the effective load acting on the contact surface, due to the varying $x$ displacements in the plate, which keeps the maximal contact pressures constant.

Potential extensions and improvements of the present study are many. A logical continuation of this work would be to investigate the alternative cases of loading: biaxial tension of the plate, pin loaded in its centre and a combination of pin and plate loading. Such a comprehensive analysis would fully characterize the behaviour of these receding contacts, which are more complex and versatile than the cases of layered structures, where all mechanical interrelations are intuitive and straightforward. The case of material dissimilarity needs to be investigated as well, because mechanical components normally used in pin joints are very often made of different materials.

Experimental verification of numerical results could, for instance, be carried out by employing the digital image correlation, which ensures good accuracy even with very small specimens. However, such an experimental method measures displacements and/or strains, so the verification of the obtained contact parameters would be indirect. In contrast, an experiment based on the photoelastic method could potentially be quite impractical if too small a model were used, since a very thin bushing would not be able to yield usable results. A sufficiently large photoelastic model would, however, yield a full-field stress distribution which could be compared easily with the FEM results and which would also directly reveal the level of agreement between both the contact angles and the contact stresses. Which of these would prove superior from the standpoint of feasibility and practicality is a research topic in its own right.

\section{REFERENCES}

[1] Johnson, K. L. (1992). Contact mechanics. Cambridge, UK: Cambridge University Press.

[2] Stippes, M., Wilson, H., \& Krull, F. N. (1962). A contact stress problem for a smooth disk in an infinite plate. Proc. of the $4^{\text {th }}$ US National Congress of Applied Mechanics, ASME, 799806.

[3] Noble, B. \& Hussain, M. A. (1969). Exact Solution for Certain Dual Series for Indentation and Inclusion Problem. International Journal of Engineering Science, 7, 1149-1161. https://doi.org/10.1016/0020-7225(69)90081-0

[4] Margetson, J. \& Morland, L. W. (1970). Separation of smooth circular inclusions from elastic and viscoelastic plates subjected to uniaxial tension. Journal of the Mechanics and Physics of Solids, 18(4), 295-309. https://doi.org/10.1016/0022-5096(70)90009-8

[5] Ghosh, S. P., Dattaguru, B., \& Rao, A. K. (1982). Photoelastic Studies on Progress of Separation in Interference Fits. Experimental Mechanics, 22(1), 8-15. https://doi.org/10.1007/BF02325696

[6] Mangalgiri, P. D., Ramamurthy, T. S., Dattaguru, B., \& Rao, A. K. (1987). Elastic analysis of pin joints in plates under some combined pin and plate loads. International Journal of Mechanical Sciences, 29(8), 577-585. https://doi.org/10.1016/0020-7403(87)90029-4

[7] Man, K. W. (1994). Contact Mechanics Using Boundary Elements. Southampton UK, Boston USA: Computational Mechanics Publications.

[8] Satish Kumar, K., Dattaguru, B., Ramamurthy, T. S., \& Raju, K. N. (1996). Elasto-plastic contact stress analysis of joints subjected to cyclic loading. Computers and Structures, 60(6), 1067-1077. https://doi.org/10.1016/0045-7949(95)00413-0

[9] de Jong, T. (1987). On the calculation of stresses in pinloaded anisotropic plates. PhD Thesis, Technishe Universiteit Delft.

[10] Murthy, A. V., Dattaguru, B., Narayana, H. V. L., \& Rao, A. K. (1991). Stress and Strength Analysis of Pin Joints in Laminated Anisotropic Plates. Composite Structures, 19(4), 299-312. https://doi.org/10.1016/0263-8223(91)90078-D

[11] Camanho, P. P. \& Matthews, F. L. (1997). Stress analysis and strength prediction of mechanically fastened joints in FRP: a review. Composites Part A: Applied Science and Manufacturing, 28(6), 529-547. https://doi.org/10.1016/S1359-835X(97)00004-3

[12] Ciavarella, M. \& Decuzzi, P. (2001). The state of stress induced by the plane frictionless cylindrical contact. I. The case of elastic similarity. International Journal of Solids and Structures, 38(26-27), 4507-4523.

[13] Ciavarella, M. \& Decuzzi, P. (2001). The state of stress induced by the plane frictionless cylindrical contact. II. The general case (elastic dissimilarity). International Journal of Solids and Structures, 38(26-27), 4525-4533. https://doi.org/10.1016/S0020-7683(00)00290-0

[14] Ho, K. C. \& Chau, K. T. (1997). An infinite plane loaded by a rivet of a different material. International Journal of Solids and Structures, 34(19), 2477-2496. https://doi.org/10.1016/S0020-7683(96)00159-X

[15] Iyer, K. (2001). Solutions for contact in pinned connections. International Journal of Solids and Structures, 38(50-51), 9133-9148. https://doi.org/10.1016/S0020-7683(01)00054-3

[16] Hou, J. P. \& Hills, D. A. (2001). Contact between a pin and a plate with a hole under interference-fit and clearance-fit conditions. Proceedings of the Institution of Mechanical Engineers. Part C: Journal of Mechanical Engineering Science, 215(6), 629-639. https://doi.org/10.1243/0954406011524009

[17] Hou, J. P. \& Hills, D. A. (2001). Interference contact between a pin and plate with a hole. Journal of Strain Analysis for 
Engineering Design, 36(5), 499-506. https://doi.org/10.1243/0309324011514656

[18] Hou, J. P. \& Hills, D. A. (2002). The effect of friction on a receding conformal contact. Proceedings of the Institution of Mechanical Engineers. Part C: Journal of Mechanical Engineering Science, 216(3), 337-342. https://doi.org/10.1243/0954406021525043

[19] Ciavarella, M., Baldini, A., Barber, J. R., \& Strozzi. A. (2006). Reduced dependence on loading parameters in almost conforming contacts. International Journal of Mechanical Sciences, 48(9), 917-925. https://doi.org/10.1016/j.jmecsci.2006.03.016

[20] Allahabadi, R. (1993). Three Dimensional Slideline Contact. MSC/NASTRAN World User's Conference.

[21] MSC.Nastran 2004 Quick Reference Guide. MSC.Software Corporation, 2003.

[22] Keer, L. M., Dundurs, J., \& Tsai, K. C. (1972). Problems Involving a Receding Contact Between a Layer and a Half Space. Transactions of the ASME - Journal of Applied Mechanics, 39(4), Series E, 1115-1120. https://doi.org/10.1115/1.3422839

[23] Tsai, K. C., Dundurs, J., \& Keer, L. M. (1974). Elastic Layer Pressed Against a Half Space. Transactions of the ASME Journal of Applied Mechanics, 41(3), Series E, 703-707. https://doi.org/10.1115/1.3423375

[24] Ahn, Y. J. \& Barber, J. (2008). Response of frictional receding contact problems to cyclic loading. International Journal of Mechanical Sciences, 50(10-11), 1519-1525. https://doi.org/10.1016/j.jimecsci.2008.08.003

[25] Rončević, B., Bakić, A., \& Kodvanj, J. (2016). Numerical and experimental analysis of a frictionless receding contact between a cylindrical indenter, layer and substrate. Transactions of FAMENA, 40(2), 1-18.

https://doi.org/10.21278/TOF.40201

\section{Contact information:}

\section{Branimir RONČEVIĆ, PhD}

(Corresponding author)

Vulkan-Nova, d.0.0.

Spinčićeva 12, 51000 Rijeka, Croatia

E-mail: broncev@yahoo.com

Ante BAKIĆ, PhD

INETEC - Institute for Nuclear Technology

Dolenica 28, 10250 Lučko, Croatia

E-mail: ante.bakic@inetec.hr

Janoš KODVANJ, PhD, Professor

Faculty of Mechanical Engineering and Naval Architecture,

University of Zagreb

Ivana Lučića 5, 10000 Zagreb, Croatia

E-mail: jkodvanj@fsb.hr 